Review Article

\title{
Is Huachansu Beneficial in Treating Advanced Non-Small-Cell Lung Cancer? Evidence from a Meta-Analysis of Its Efficacy Combined with Chemotherapy
}

\author{
Bingduo Zhou, ${ }^{1}$ Fengying Wu, ${ }^{2}$ Lin Yuan, ${ }^{3}$ Zhulei Miao, ${ }^{4}$ and Shengliang Zhu ${ }^{1}$ \\ ${ }^{1}$ Department of Gastroenterology, Yueyang Hospital of Integrated Traditional Chinese and Western Medicine, \\ Shanghai University of Traditional Chinese Medicine, Shanghai 200437, China \\ ${ }^{2}$ Department of Oncology, Shanghai Pulmonary Hospital, Tongji University School of Medicine, Shanghai 200433, China \\ ${ }^{3}$ Science and Technology Experimental Center, Shanghai University of Traditional Chinese Medicine, Shanghai 201203, China \\ ${ }^{4}$ Department of Immunology and Pathogen Biology, School of Basic Medicine, Shanghai University of Traditional Chinese Medicine, \\ Shanghai 201203, China
}

Correspondence should be addressed to Zhulei Miao; mzhulei@yeah.net and Shengliang Zhu; zhushengliang999@163.com

Received 29 January 2015; Accepted 24 June 2015

Academic Editor: José L. Ríos

Copyright (C) 2015 Bingduo Zhou et al. This is an open access article distributed under the Creative Commons Attribution License, which permits unrestricted use, distribution, and reproduction in any medium, provided the original work is properly cited.

Background. Huachansu, the sterilized water extract of Bufo bufo gargarizans toad skin, is used in China to alleviate the side-effects and enhance the therapeutic effect of chemotherapy in advanced non-small-cell lung cancer (NSCLC). We conducted a metaanalysis to assess Huachansu's efficacy. Methods. We extensively searched electronic databases (CENTRAL, EMBASE, MEDLINE, CBM, Cochrane Library, CNKI, CEBM, WFDP, CSCD, CSTD, and IPA) for randomized controlled trials containing Huachansu plus chemotherapy as the test group and chemotherapy as the control group. Seventeen trials were selected based on the selection criteria. The pooled relative ratio (RR) of indicators with $95 \%$ confidence interval (95\% CI) was calculated for efficacy evaluation. Results. The meta-analysis demonstrated a statistically significant improvement in objective tumor response, one-year survival, Karnofsky performance status, pain relief, and alleviation of severe side-effects (nausea and vomiting, leukocytopenia) in the test group as compared to the control group, but no significant difference in thrombocytopenia. Conclusions. This study demonstrated the efficacy of Huachansu combined with chemotherapy in the treatment of advanced NSCLC. However, limitations exist and high-quality trials are needed for further verification.

\section{Introduction}

Non-small-cell lung cancer (NSCLC) is the most common type of lung cancer, which is difficult to diagnose in early stages. Patients diagnosed with advanced NSCLC are often unable to undergo surgery, thus reducing the survival time [1]. So far, chemotherapy is typically used for the treatment of advanced NSCLC. However, chemotherapy not only kills tumor cells but also causes several side-effects. Many patients have to endure great discomfort to prolong their lives for a limited time. So providing efficacious therapy while improving the patient's quality of life is the primary concern of clinicians when selecting appropriate regimens.
Huachansu (Cinobufacini) is the sterilized water extract of Bufo bufo gargarizans toad skin [2], a small amphibian animal that is widely distributed in China and has survived on the earth for millions of years. During this time, the toad's skin developed the ability to produce various active ingredients to protect itself from adverse environmental factors [3]. These ingredients include alkaloids, peptides, and steroids, which inhibit the growth of fungi, bacteria, and viruses, stimulate nerves, and induce anesthesia (a powerful weapon against predators) [4]. Moreover, other ingredients have been identified, which kill cancer cells, induce apoptosis, prevent angiogenesis, and enhance the immune system $[5,6]$, which make the skin of Bufo bufo gargarizans a valuable source 
of traditional Chinese medicine (TCM) [7, 8]. The Chinese Food and Drug Administration approved Huachansu in 1991 for the treatment of chronic HBV infection and cancers (predominantly as standard-of-care monotherapy for pancreatic and hepatobiliary malignancies) [9].

In order to alleviate the side-effects of chemotherapy and enhance therapeutic efficacy in advanced NSCLC, several clinical studies have been conducted using Huachansu combined with chemotherapy to observe whether this would be beneficial for patients. But due to the limited scale of these studies, certain results remain inconsistent. Therefore, we performed a meta-analysis by pooling the randomized clinical trials to evaluate the efficacy of this complementary therapy.

\section{Material and Methods}

2.1. Search Strategy. Clinical trials were retrieved from the Cochrane Central Register of Controlled Trials (CENTRAL); EMBASE; MEDLINE; Cochrane Library; Chinese Biological Medicine Database (CBM); China National Knowledge Infra-Structure Database (CNKI); Chinese Evidence-Based Medicine Database (CEBM); Wan Fang Digital Periodicals Database (WFDP); China Science Citation Database (CSCD); Chinese Science Technology Document Database (CSTD); and the International Pharmaceutical Abstracts (IPA), from their inception to January 2015. The retrieval terms used were as follows: (non-small-cell lung cancer OR non-small-cell lung carcinoma OR NSCLC OR squamous cell lung carcinoma OR large cell lung carcinoma OR lung adenocarcinoma) AND (Huachansu OR Hua chan su OR Cinobufacini).

2.2. Study Selection. Studies that met the following criteria were included: (1) type of studies: clinical randomized controlled trials (RCTs); (2) participants: age $\geq 18$ years with pathology and CT diagnosis of NSCLC in stage III or IV with detectable solid tumor and KPS $\geq 60$; (3) type of intervention: treatment with Huachansu combined with chemotherapy as the test group; (4) type of outcome: reports with at least one of the following indicators: objective tumor response, one-year survival, quality of life (KPS scale), pain relief, major drug adverse reactions, or the data necessary to calculate them. Clinical trials were excluded if they did not meet the above criteria. The following types of studies were also excluded: (1) treatment without chemotherapy as the control group; (2) nonoriginal or duplicated publications; (3) patients with other serious illnesses.

2.3. Quality Assessment and Data Extraction. All reports were independently reviewed by two reviewers (BD Zhou and FY $\mathrm{Wu}$ ) to evaluate their quality, decide which study would meet the eligibility criteria, and extract the data needed: author names, year of publication, study type, patient information, details of the treatment, and outcome. Any disagreements were resolved through discussion or by a third reviewer.
2.4. Meta-Analysis. The STATA (version 11.0) was used for data analysis. Relative ratio (RR) and 95\% confidence intervals $(\mathrm{CI})$ were calculated. $p<0.05$ was considered to be statistically significant. To determine whether the randomeffects or fixed-effects model should be used, the $I^{2}$ was used to estimate heterogeneity (random-effects model when $I^{2} \geq$ $50 \%$ or fixed-effects model when $I^{2}<50 \%$ ) [27].

The indicators for evaluation of therapeutic efficacy were as follows: (1) objective tumor response; (2) one-year survival; (3) improved performance status; (4) pain relief; (5) symptoms of severe side-effects caused by chemotherapy (nausea and vomiting, leukocytopenia, and thrombocytopenia).

\section{Results}

3.1. Description of Enrolled Studies. In this study, seventeen trials were selected based on selection criteria. A total of 80 publications were originally identified, of which 63 studies were excluded and 17 studies were finally included based on the selection criteria with 1142 enrolled patients (578 in the test group and 564 in the control group) [10-26]. The selection process and reasons for exclusion are described in Figure 1, and the characteristics of the included studies are presented in Table 1.

All 17 studies mentioned random allocation, but only six studies described random allocation methods. No study described the blind method and allocation concealment. All patients recruited in these studies were diagnosed at stages III to IV NSCLC TNM. These patients were given an overall physical assessment before treatment, including age, quality of life score, and stage of disease. No significant differences between the baseline data were reported and all patients were estimated with life expectancy $\geq 3$ months. Different combination chemotherapies were used for treatment: vinorelbine plus cisplatin (NP), docetaxel plus cisplatin (DC), paclitaxel plus cisplatin (TP), gemcitabine plus cisplatin (GP), etoposide plus cisplatin (EP), vinorelbine plus carboplatin (NC), vinorelbine plus ifosfamide (NI), and pemetrexed plus cisplatin. The methodological quality was evaluated using the Jadad scale according to the description in these reports on randomization, blinding method, withdrawals/dropouts, and allocation concealment, which was presented in Table 2 .

3.2. Objective Tumor Response Results. Thirteen trials reported the number of treated patients with complete response (CR), partial response (PR), stable disease (SD), and progressive disease (PD) in each group, based on the WHO scale. The objective tumor response was determined by the number of patients with CR plus PR. Meta-analysis showed a significant increase in the number of patients with CR plus PR in the Huachansu plus chemotherapy test group ( $R R=1.379,95 \%$ CI, 1.190-1.599, $p<0.0001,879$ patients). A fixed-effects model was used since heterogeneity was absent $\left(I^{2}=0 \%\right.$, $p=0.989)$ (Figure 2).

3.3. One-Year Survival Results. Four trials reported the number of treated patients who survived $>1$ year in each group. Meta-analysis showed a significant increase in the number of 


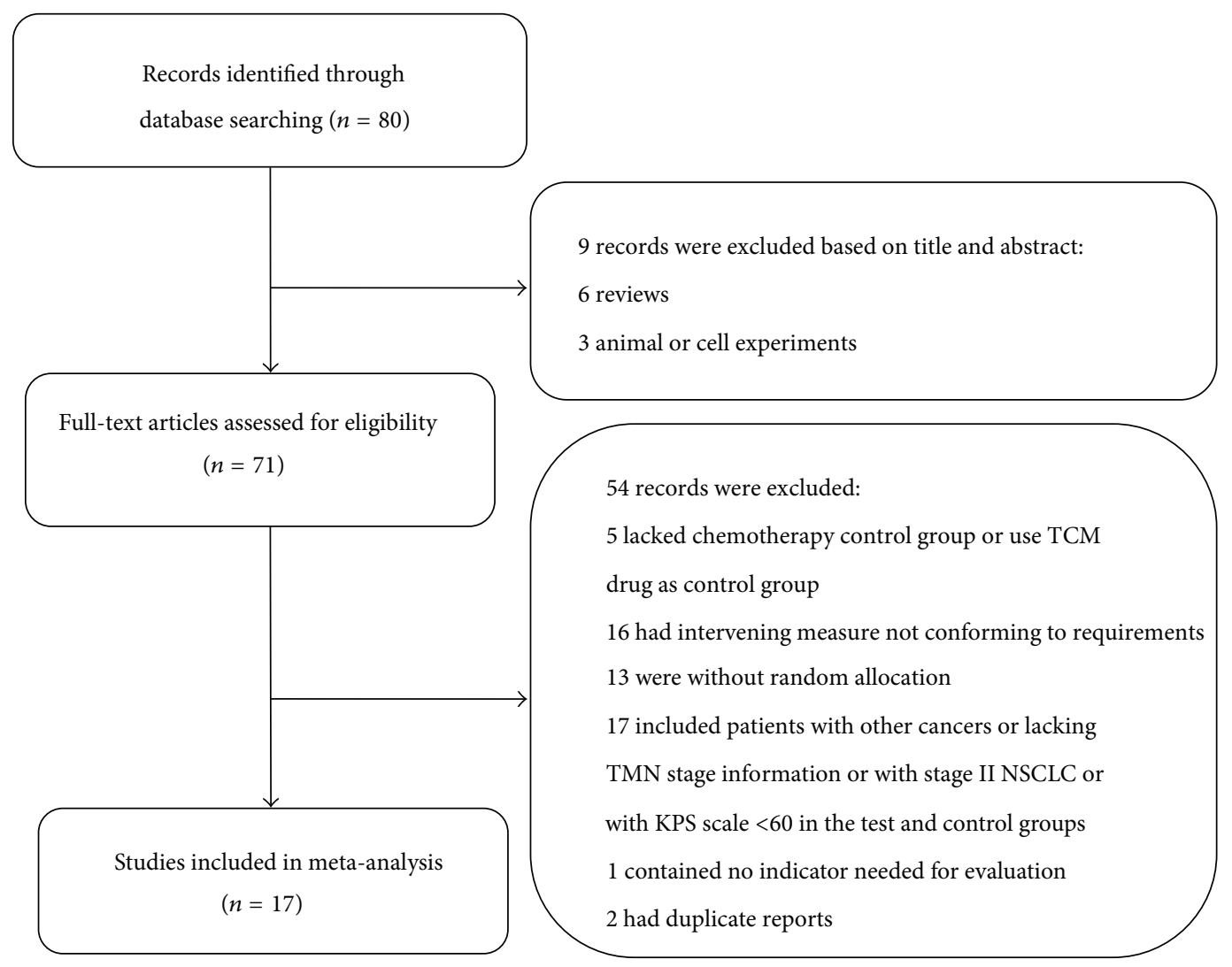

FIGURE 1: Flow chart of study selection.

\begin{tabular}{|c|c|c|c|}
\hline Study ID & & RR $(95 \%$ CI $)$ & Weight (\%) \\
\hline Bao et al. (2011) & $\rightarrow \quad 1$ & $1.22(0.80,1.86)$ & 12.30 \\
\hline Сao (2009) & & $1.25(0.59,2.64)$ & 4.84 \\
\hline Ding (2011) & 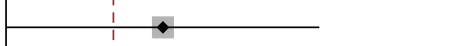 & $1.60(1.00,2.56)$ & 9.07 \\
\hline Jing (2007) & 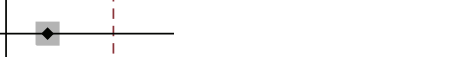 & $1.13(0.78,1.65)$ & 10.97 \\
\hline Li et al. (2010) & 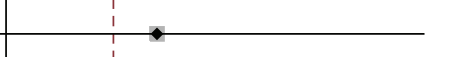 & $1.57(0.71,3.50)$ & 4.23 \\
\hline Liu (2014) & & $1.51(0.79,2.91)$ & 6.41 \\
\hline Miao et al. (2007) & & $1.64(1.00,2.67)$ & 8.97 \\
\hline Miao et al. (2014) & 1 & $1.60(0.87,2.94)$ & 6.05 \\
\hline Qi (2011) & & $1.42(0.83,2.43)$ & 7.26 \\
\hline Wang (2006) & $\rightarrow$ & $1.13(0.72,1.75)$ & 9.68 \\
\hline Xiong and Li (2005) & $-i$ & $1.37(0.83,2.26)$ & 8.12 \\
\hline Yang and Xi (2006) & & $1.57(0.71,3.50)$ & 4.23 \\
\hline Yu et al. (2012) & 4 & $1.31(0.77,2.22)$ & 7.86 \\
\hline Overall $\left(I^{2}=0.0 \%, p=0.989\right)$ & & $1.38(1.19,1.60)$ & 100.00 \\
\hline & 1 & & \\
\hline 0.286 & 3.5 & & \\
\hline
\end{tabular}

FIGURE 2: Forest-plot of objective tumor response. 


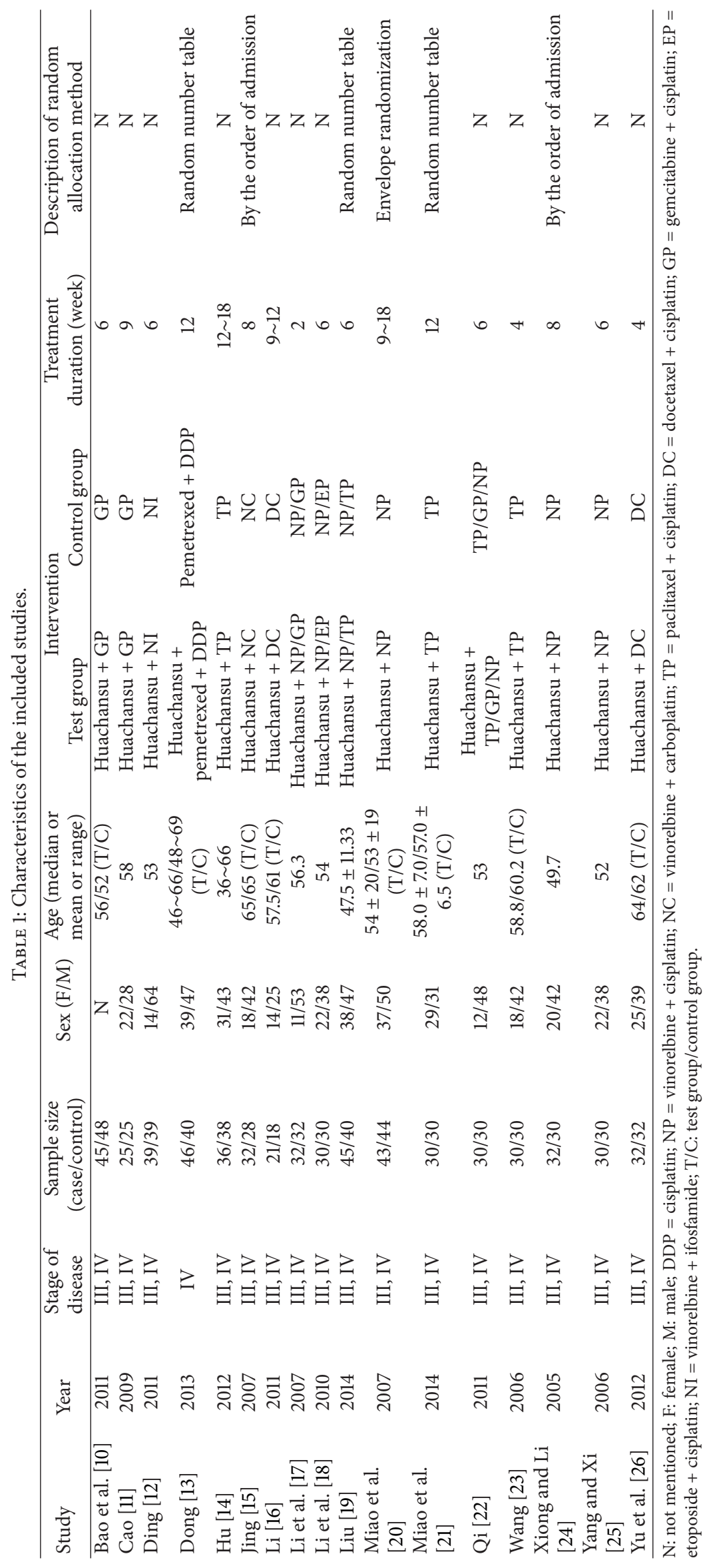




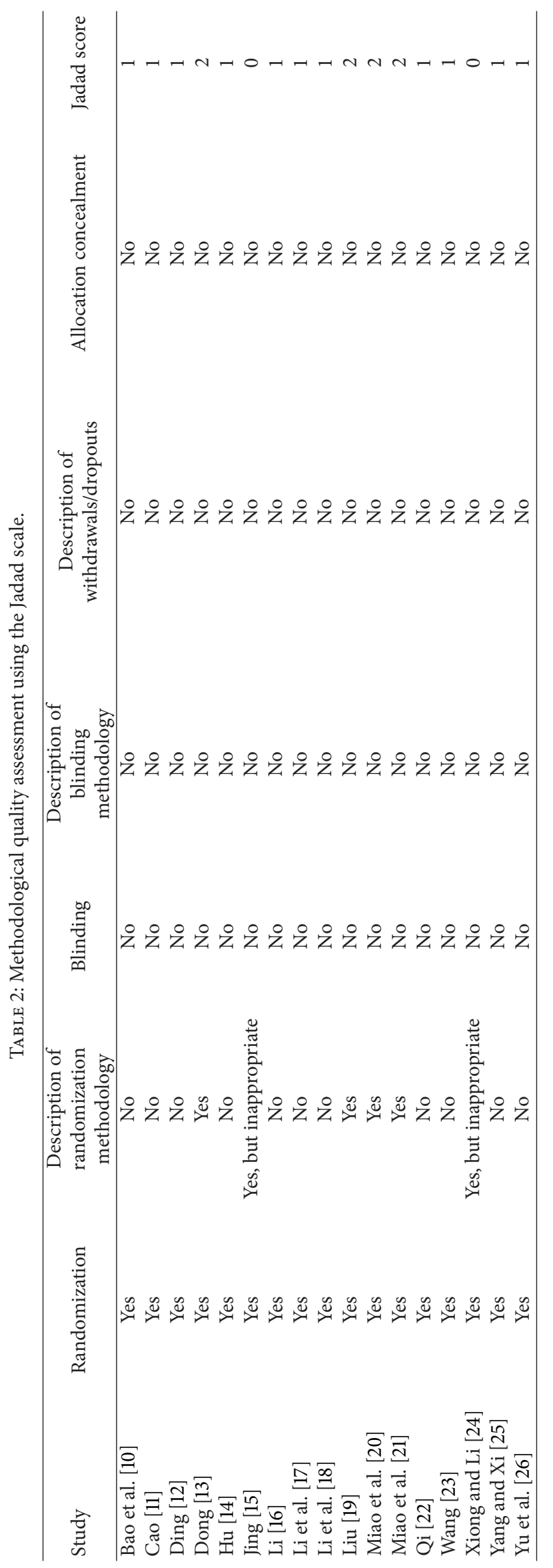




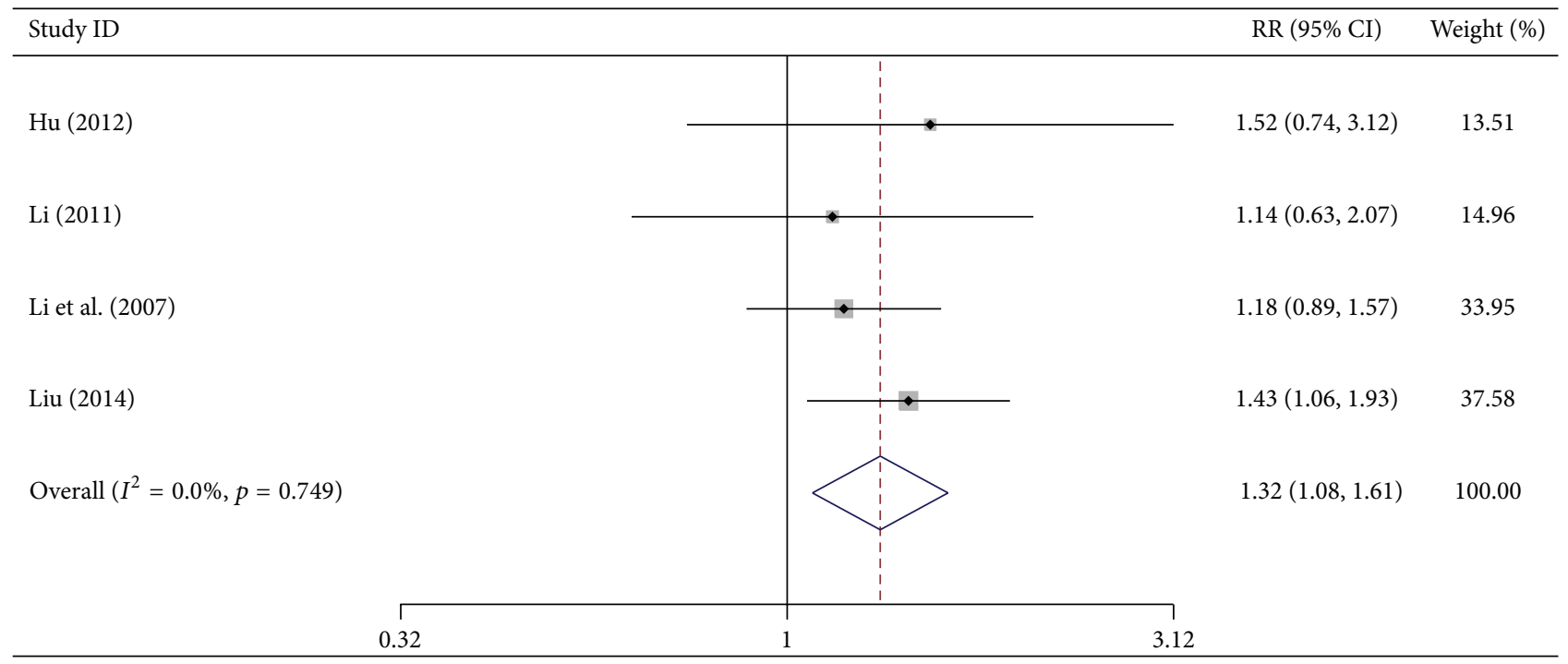

FIGURE 3: Forest-plot of one-year survival.

TABLE 3: Result of sensitivity analysis of the studies belonging to different indicator groups.

\begin{tabular}{|c|c|c|c|c|c|c|c|}
\hline \multirow{2}{*}{ Indicator } & \multirow{2}{*}{ Number of trials } & \multicolumn{3}{|c|}{ Fixed-effects model } & \multicolumn{3}{|c|}{ Random-effects model } \\
\hline & & $\mathrm{RR}$ & $95 \% \mathrm{CI}$ & $p$ value & $\mathrm{RR}$ & $95 \% \mathrm{CI}$ & $p$ value \\
\hline Objective tumor response & 13 & 1.379 & $1.190-1.599$ & $p<0.0001$ & 1.351 & $1.168-1.562$ & $p<0.0001$ \\
\hline One-year survival & 4 & 1.316 & $1.077-1.607$ & $p=0.007$ & 1.293 & $1.071-1.561$ & $p=0.008$ \\
\hline Performance Status & 9 & 1.397 & $1.185-1.648$ & $p<0.0001$ & 1.351 & $1.160-1.572$ & $p<0.0001$ \\
\hline Pain relief & 4 & 1.640 & $1.293-2.080$ & $p<0.0001$ & 1.604 & $1.273-2.022$ & $p<0.0001$ \\
\hline Nausea and vomiting & 7 & 0.523 & $0.333-0.822$ & $p=0.005$ & 0.538 & $0.297-0.974$ & $p=0.041$ \\
\hline Leukocytopenia & 9 & 0.644 & $0.473-0.876$ & $p=0.005$ & 0.638 & $0.468-0.871$ & $p=0.005$ \\
\hline Thrombocytopenia & 5 & 0.593 & $0.334-1.054$ & $p=0.075$ & 0.611 & $0.344-1.083$ & $p=0.092$ \\
\hline
\end{tabular}

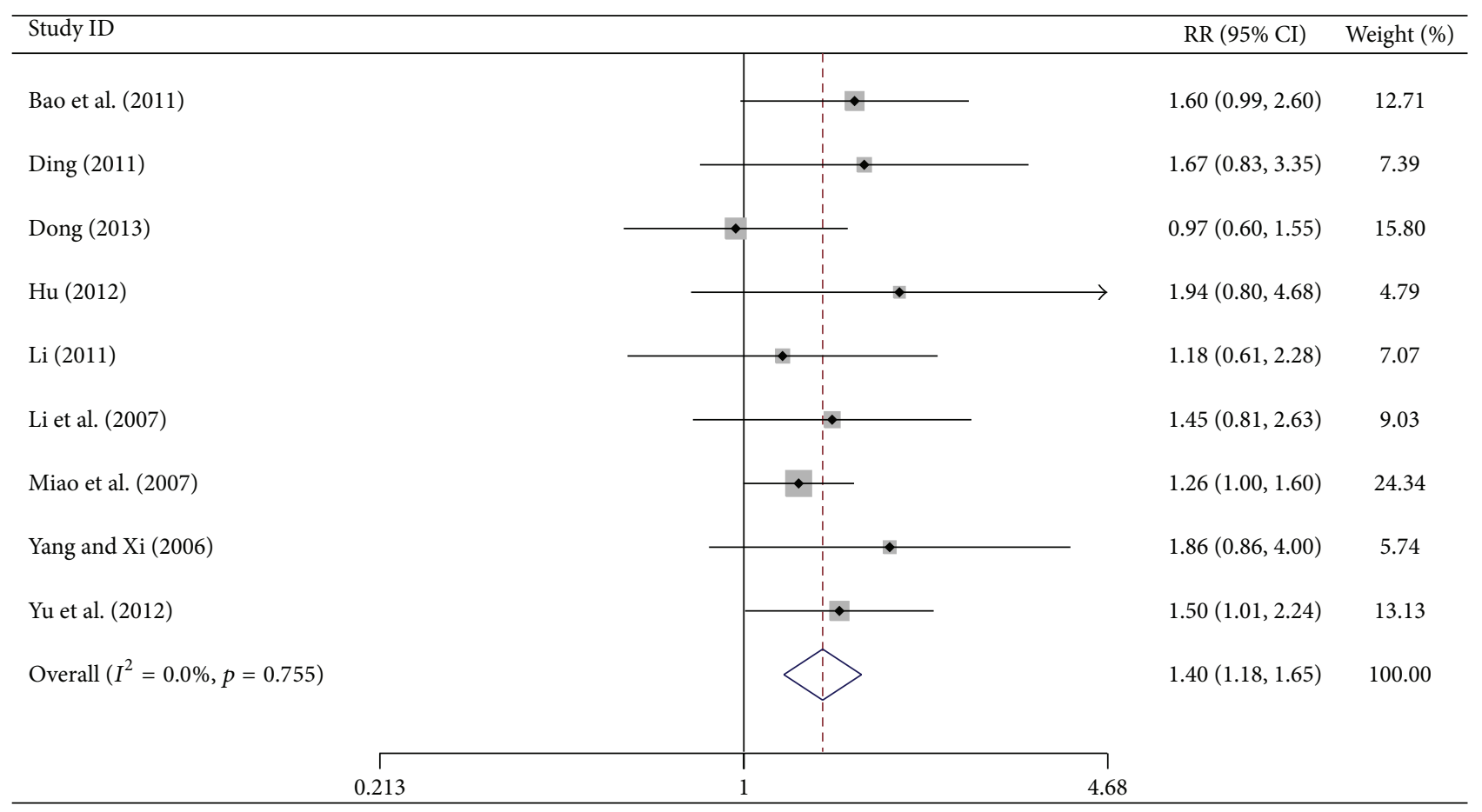

FIGURE 4: Forest-plot of improved Karnofsky performance status. 


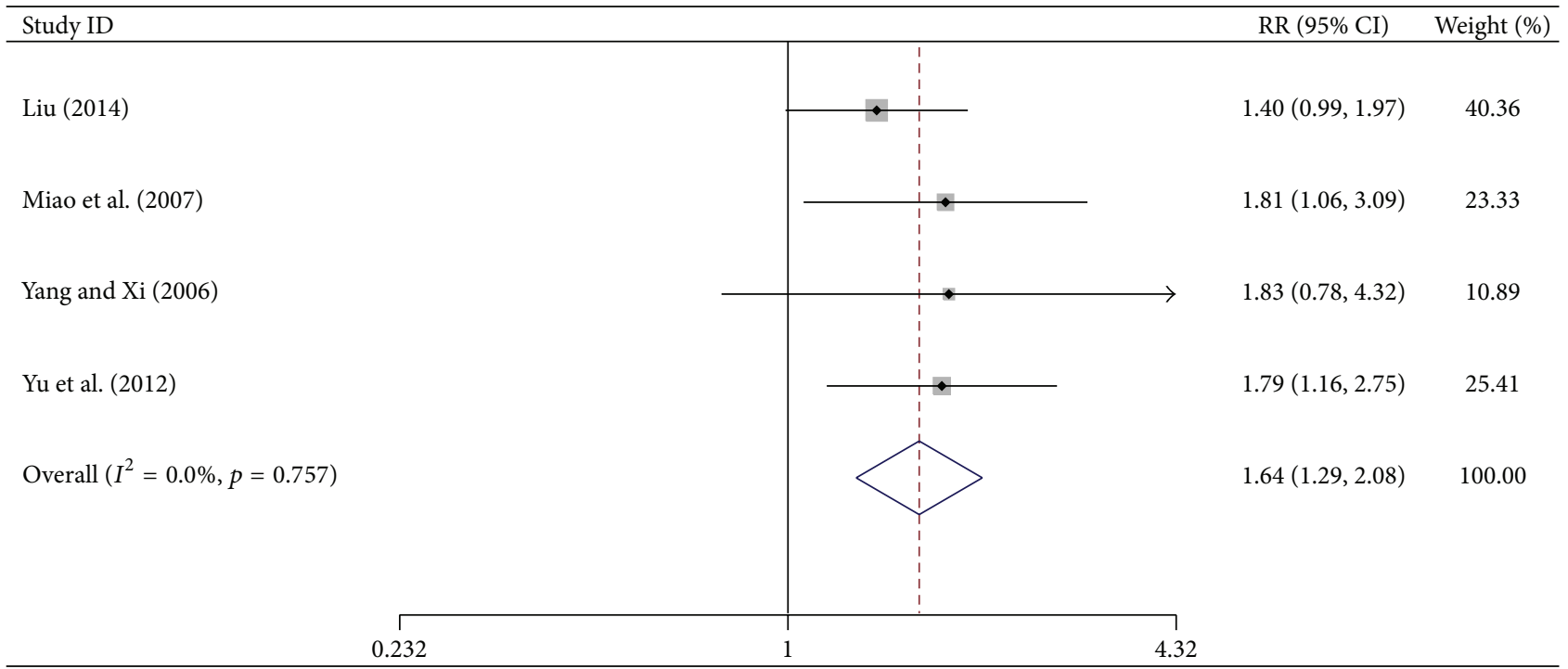

FIGURE 5: Forest-plot of pain relief.

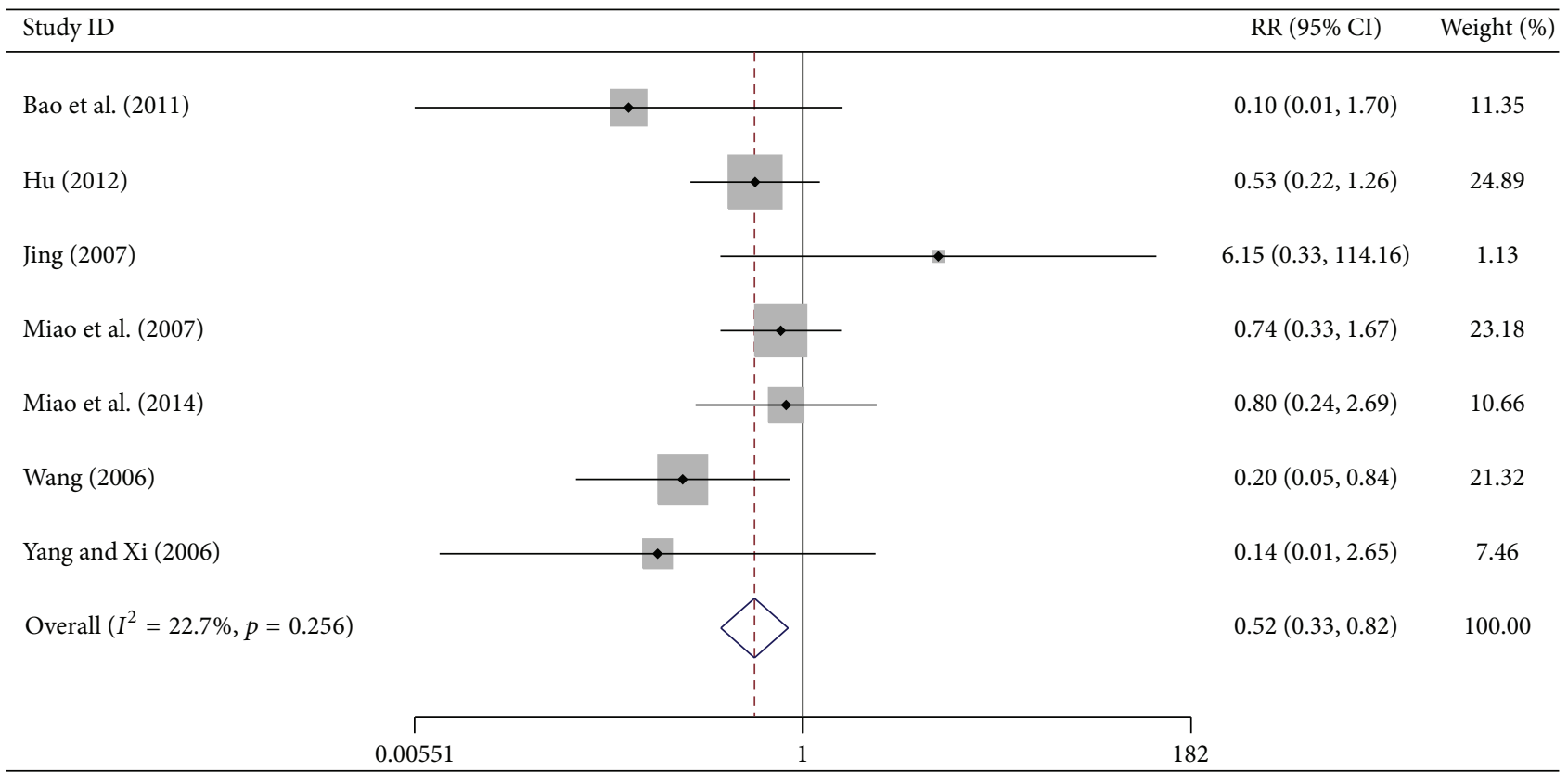

FIGURE 6: Forest-plot of nausea and vomiting at the grade of III IV.

patients surviving $>$ one year in the test group as compared to the control group $(\mathrm{RR}=1.316,95 \% \mathrm{CI}, 1.077-1.607, p=$ $0.007,262$ patients). As heterogeneity was absent $\left(I^{2}=0 \%\right.$, $p=0.749$ ), a fixed-effects model was used (Figure 3).

3.4. Performance Status Results. Nine trials reported the number of patients with improved status (an increase of $\geq 10$ points), stable status (an increase or decrease of $<10$ points), and decline status (a decrease of $\geq 10$ points) in each group based on the Karnofsky performance scale (KPS). Meta-analysis showed a significant increase in the number of patients with improved status in the test group
( $\mathrm{RR}=1.397,95 \% \mathrm{CI}, 1.185-1.648, p<0.0001,645$ patients). Since heterogeneity was absent $\left(I^{2}=0 \%, p=0.755\right)$, a fixedeffects model was used (Figure 4).

3.5. Pain Relieving Effects. Four trials reported the number of patients with pain relief after treatment. The degree of pain was assessed with the WHO rating scale. Meta-analysis showed a significant increase in the number of patients with complete plus partial pain relief in the test group $(\mathrm{RR}=$ $1.64,95 \%$ CI, 1.293-2.080, $p<0.0001,296$ patients). Since heterogeneity was absent $\left(I^{2}=0 \%, p=0.757\right)$, a fixed-effects model was used (Figure 5). 


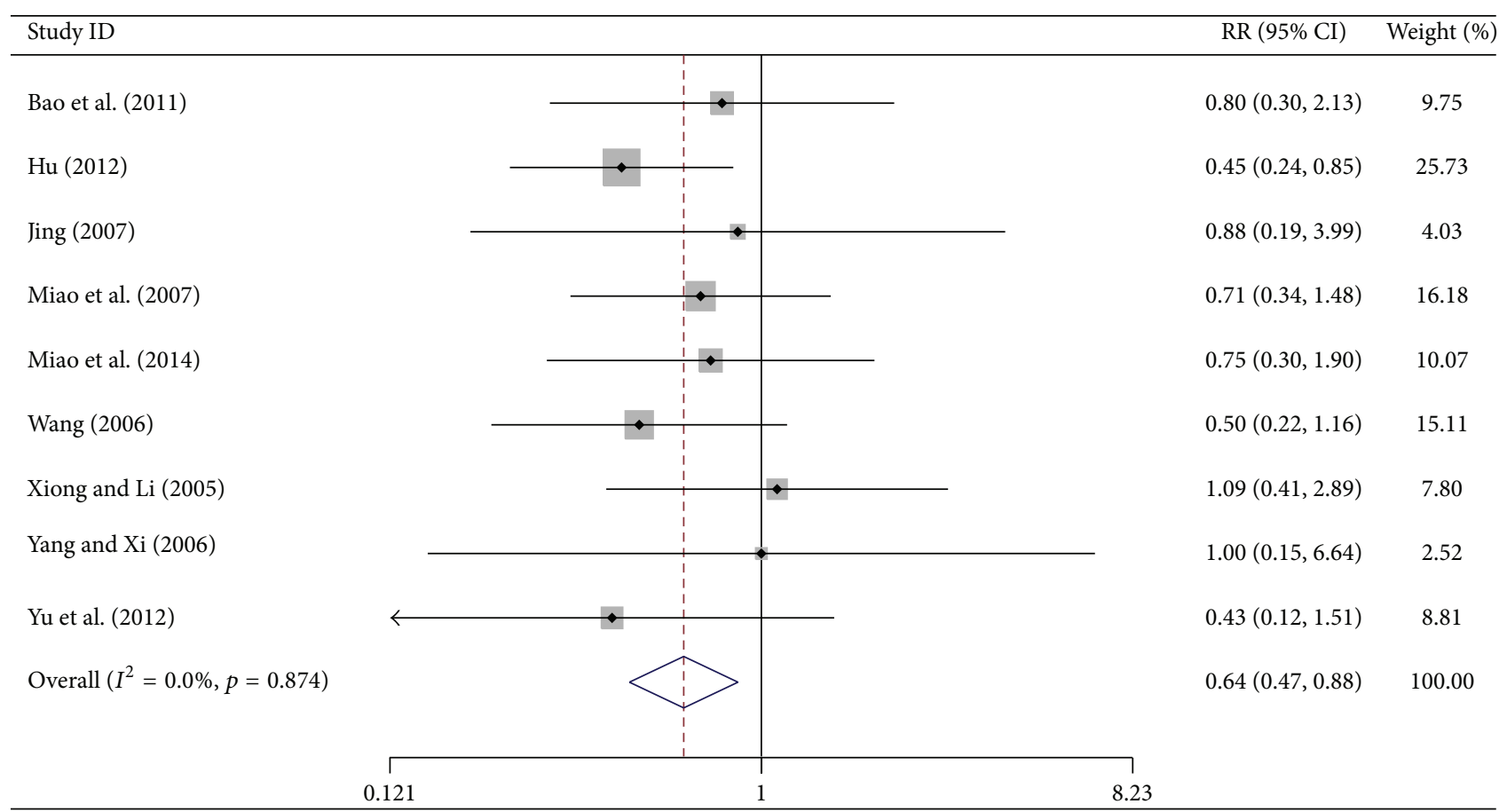

FIGURE 7: Forest-plot of leukocytopenia at the grade of III IV.

\begin{tabular}{|c|c|c|c|}
\hline Study ID & & RR $(95 \%$ CI $)$ & Weight (\%) \\
\hline Bao et al. (2011) & & $0.43(0.09,2.09)$ & 18.41 \\
\hline $\mathrm{Hu}(2012)$ & & $0.53(0.10,2.71)$ & 14.81 \\
\hline Jing (2007) & & $0.58(0.10,3.24)$ & 12.18 \\
\hline Miao et al. (2007) & & $0.71(0.34,1.48)$ & 48.90 \\
\hline Yang and Xi (2006) & & $0.33(0.01,7.87)$ & 5.71 \\
\hline Overall $\left(I^{2}=0.0 \%, p=0.970\right)$ & & $0.59(0.33,1.05)$ & 100.00 \\
\hline 0.0141 & 70.8 & & \\
\hline
\end{tabular}

FIGURE 8: Forest-plot of thrombocytopenia at the grade of III IV.

3.6. Severe Chemotherapy Toxicity Results. Severe chemotherapy toxicities such as gastrointestinal side-effects and myelosuppression are among the most important causes for patients' failure to treatment adherence. Seven trials reported the number of cases with nausea and vomiting of grade III or IV (WHO scale). Meta-analysis showed a significant reduction in the number of cases in the test group as compared to the control group $(\mathrm{RR}=0.523,95 \% \mathrm{CI}, 0.333-$ $0.822, p=0.005,494$ patients). A fixed-effects model was used, as the heterogeneity was not significant $\left(I^{2}=22.7 \%\right.$, $p=0.256$ ) (Figure 6). Nine trials reported the number of patients with leukocytopenia of grade III or IV (WHO scale). Meta-analysis showed a significant decrease in the number of patients with grade III or IV leukocytopenia in the test group $(\mathrm{RR}=0.644,95 \% \mathrm{CI}, 0.473-0.876, p=$ $0.005,620$ patients). Since no heterogeneity was observed $\left(I^{2}=0 \%, p=0.874\right)$, a fixed-effects model was used (Figure 7). Five trials reported the number of treated patients with thrombocytopenia of grade III or IV (WHO scale). Although a trend of improvement was observed in the test group, there was no statistically significant difference between the two groups $(\mathrm{RR}=0.593,95 \% \mathrm{CI}, 0.334-1.054, p=0.075$, 


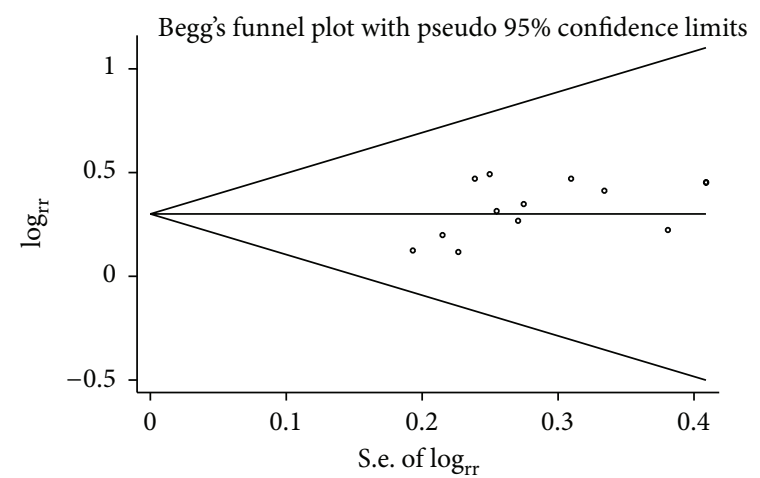

(a)

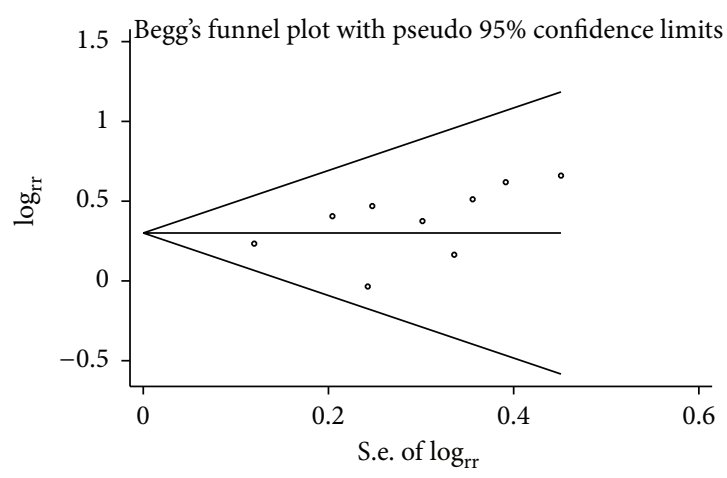

(c)

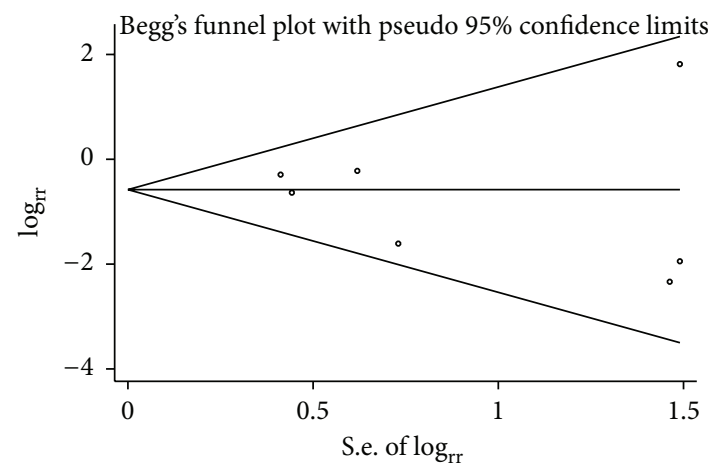

(e)

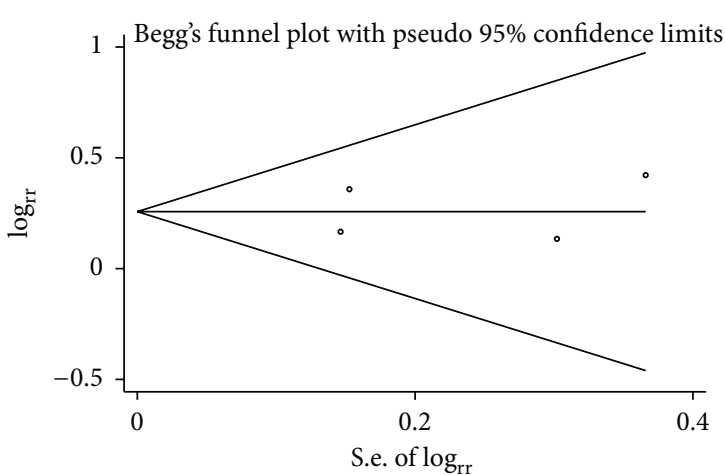

(b)

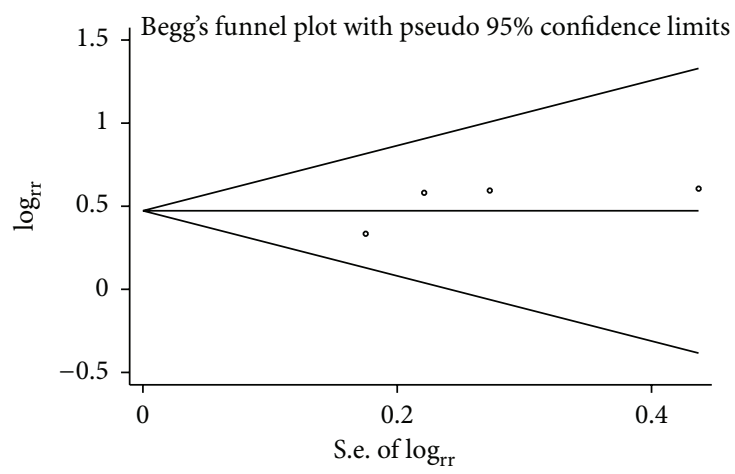

(d)

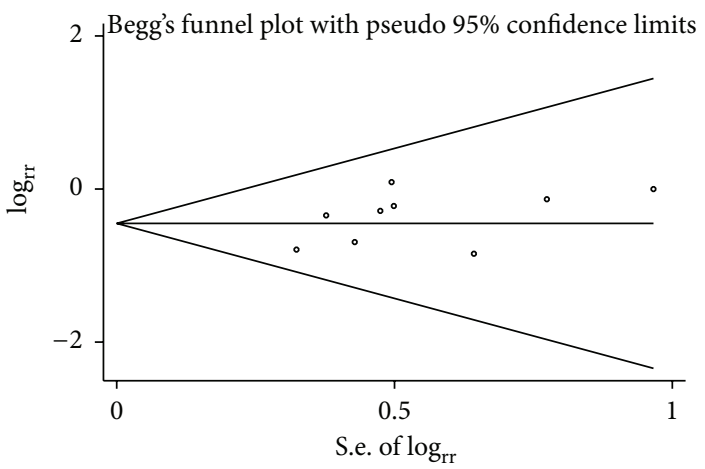

(f)

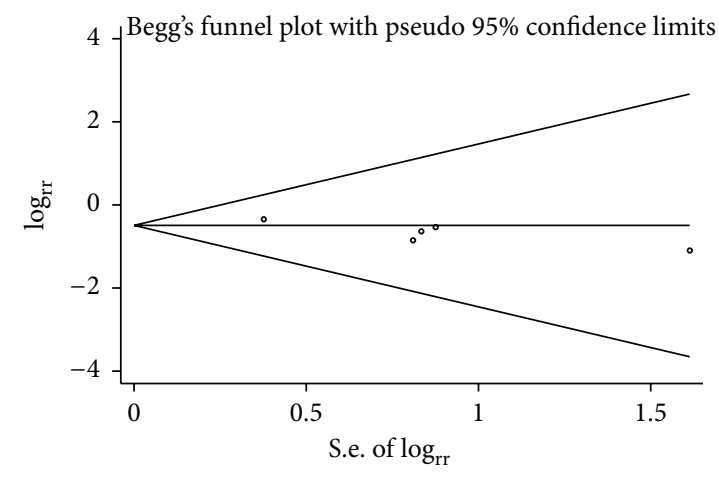

(g)

FIGURE 9: Funnel plots of the studies belong to different groups: (a) objective tumor response; (b) one-year survival; (c) improved Karnofsky performance status; (d) pain relief; (e) nausea and vomiting; (f) leukocytopenia; (g) thrombocytopenia. 
TABLE 4: Result of Begg's and Egger's test for publication bias of the studies belonging to different indicator groups.

\begin{tabular}{lccc}
\hline Indicator & \multirow{2}{*}{ Number of trials } & \multicolumn{2}{c}{ Publication bias } \\
\hline Objective tumor response & & $p$ value (Begg's) & 0.143 \\
One-year survival & 13 & 0.734 & 0.888 \\
Performance status & 4 & 0.076 & 0.19 \\
Pain relief & 9 & 1.000 & 0.300 \\
Nausea and vomiting & 4 & 0.764 & 0.603 \\
Leukocytopenia & 7 & 0.175 & 0.212 \\
Thrombocytopenia & 9 & 0.806 & 0.036 \\
\hline
\end{tabular}

374 patients). As heterogeneity was absent $\left(I^{2}=0 \%, p=\right.$ 0.970), a fixed-effects model was used (Figure 8).

3.7. Sensitivity Analysis Results. The fixed-effects and random-effects models were used to perform sensitivity analysis. Though the $p$ value increased for certain indicators when using the random-effects model, the outcomes did not change. The results are displayed in Table 3.

3.8. Publication Bias Analysis. Begg's funnel plot and Egger's linear regression test were used to evaluate the publication bias. The result of Egger's test suggested a publication bias among the studies within the group of thrombocytopenia $(p=0.036)$. No bias was observed in the other groups. The results are displayed in Figure 9 and Table 4.

\section{Discussion}

So far Huachansu has been used mainly in China, and most of the clinical trials were performed by separate hospitals with a small sample size. The number of patients included in most trials was $<100$. So certain results were not very convincing. For example, five studies claimed no statistically significant improvement in the objective tumor response in the test group. A meta-analysis offered the unique advantage of merging all these outcomes to get a more comprehensive and accurate evaluation of such complementary therapy.

This meta-analysis showed that Huachansu can increase the objective tumor response and one-year survival, improve performance status, alleviate severe chemotherapy sideeffects, and relieve cancer pain, which may be attributed to the various ingredients in Huachansu, which is just like a troop equipped with multiple weapons. So far, more than 30 ingredients have been identified in the skin secretion of Bufo bufo gargarizans and the major functional ingredients include cardiac glycosides (Cinobufagin, Resibufogenin, Bufalin, etc.) and indole alkaloids (Bufotenine, Cinobufotenine, Serotonin, etc.). Experiments have shown that Cinobufagin, Resibufogenin, and Bufalin strongly inhibit tumor cells, and Bufotenine, Cinobufotenine, and Serotonin can act on the neural system $[5,28]$. Therefore, the clinical effects of Huachansu correspond well with the physiological activities of these ingredients.

\section{Conclusions}

In summary, this meta-analysis suggests that Huachansu, as a natural medicine containing many active ingredients, can be a promising supplement to routine chemotherapy in treating advanced NSCLC. However a major limitation of this study is the low methodological quality of the included reports. Therefore, more high-quality trials are needed in the future to validate these findings. Besides, to avoid publication bias, the likelihood of publication of trials should in no way depend on whether the results were positive or negative.

\section{Conflict of Interests}

The authors declare no conflict of interests for publication of this paper.

\section{Authors' Contribution}

Bingduo Zhou and Fengying $\mathrm{Wu}$ are equal contributors.

\section{Acknowledgments}

This study was supported by the National Natural Science Fund of China (81302929), National Natural Science Fund of China (81402381), and Innovation Program of Shanghai Municipal Education Commission (14YZ065).

\section{References}

[1] J. R. Molina, P. Yang, S. D. Cassivi, S. E. Schild, and A. A. Adjei, "Non-small cell lung cancer: epidemiology, risk factors, treatment, and survivorship," Mayo Clinic Proceedings, vol. 83, no. 5, pp. 584-594, 2008.

[2] H. Zhao, X. Wu, H. Wang et al., "Qualitative and quantitative analysis of cinobufacini injection using rapid separation liquid chromatography coupled with quadrupole-time-of-flight mass spectrometry and HPLC-photodiode array detection, a feasible strategy for the quality control of Chinese medicine injections," Journal of Separation Science, vol. 36, no. 3, pp. 492-502, 2013.

[3] B. T. Clarke, "The natural history of amphibian skin secretions, their normal functioning and potential medical applications," Biological Reviews of the Cambridge Philosophical Society, vol. 72, no. 3, pp. 365-379, 1997.

[4] D.-L. Wang, F.-H. Qi, W. Tang, and F.-S. Wang, "Chemical constituents and bioactivities of the skin of Bufo bufo gargarizans 
cantor," Chemistry and Biodiversity, vol. 8, no. 4, pp. 559-567, 2011.

[5] F. Qi, A. Li, Y. Inagaki et al., "Antitumor activity of extracts and compounds from the skin of the toad Bufo bufo gargarizans Cantor," International Immunopharmacology, vol. 11, no. 3, pp. 342-349, 2011.

[6] L. Liu, B.-A. Chen, and S.-K. Qin, "Anti-angiogenesis effect of arsenic trioxide plus cinobufacin on human hepatocarcinoma transplantation model nude mice," Chinese Journal of Integrated Traditional and Western Medicine, vol. 31, no. 1, pp. 67-72, 2011.

[7] Z. L. Miao, K. Zhang, M. Z. Yang, and X. J. Zhou, "Studies on anti-tumor and enhancing immunity activity of toad coat," China Journal of Chinese Materia Medica, vol. 35, no. 2, pp. 211214, 2010.

[8] Z. L. Miao, K. Zhang, Q. M. Bai, M. Z. Yang, and X. J. Zhou, "Textual research on Chantui-a new official Part of Bufo bufo gargarizans Cantor," Lishizhen Medicine and Materia Medica Research, vol. 17, no. 11, pp. 2323-2324, 2006.

[9] Z. Meng, C. R. Garrett, Y. Shen et al., "Prospective randomised evaluation of traditional Chinese medicine combined with chemotherapy: a randomised phase II study of wild toad extract plus gemcitabine in patients with advanced pancreatic adenocarcinomas," British Journal of Cancer, vol. 107, no. 3, pp. 411-416, 2012.

[10] W. L. Bao, Y. J. Zhang, and Y. Sun, "Clinical study of the Huachansu combined with chemotherapy in treatment of advanced non-small cell lung cancer," Zhejiang Journal of Traditional Chinese Medicine, vol. 46, no. 7, pp. 478-479, 2011.

[11] C. Y. Cao, "Clinical study of the Huachansu combined with NP regimen in treatment of advanced non-small cell lung cancer," The Journal of Medical Theory and Practice, vol. 22, no. 8, pp. 935-936, 2009.

[12] Y. J. Ding, "Clinical study of the Huachansu combined with NI regimen in treatment of advanced non-small cell lung cancer," Shandong Medical Journal, vol. 51, no. 9, pp. 76-77, 2011.

[13] J. Dong, "Clinical observation of the Huachansu combined with NP regimen in treatment of advanced non-small cell lung cancer," Chinese Journal of Traditional Medical Science and Technology, vol. 20, no. 3, p. 304, 2013.

[14] Z.-H. Hu, "Clinical observation of cinobufacini injection combined with TP regimen for advanced non-small-cell lung cancer," China Pharmacy, vol. 23, no. 16, pp. 1507-1510, 2012.

[15] E. Jing, "Clinical study of the Huachansu combined with chemotherapy in treatment of advanced non-small cell lung cancer," Chinese Remedies \& Clinics, vol. 7, no. 5, pp. 380-381, 2007.

[16] J. Li, "Clinical observation of the Huachansu combined with chemotherapy in treatment of advanced non-small cell lung cancer," Chinese Journal of Medical Guide, vol. 13, no. 11, pp. 1907-1908, 2011.

[17] M. Li, C. Xu, and X. Liu, "Clinical observation of Hua Chan Su injection in the adjuvant therapy for 64 patients with non-small cell lung cancer," Tumor, vol. 27, no. 8, pp. 666-668, 2007.

[18] X. Y. Li, X. Y. Zhang, Y. Q. Yan, L. L. Pan, and Y. J. Wang, "The effect of the combination program of Huachansu and chemotherapy on patients with non-small-cell carcinoma," China Medcine, vol. 5, no. 8, pp. 691-692, 2010.

[19] B. D. Liu, "Observe the clinical effect of cinobufacin capsules in the treatment of small cell lung cancer," Journal of Clinical and Experimental Medicine, vol. 13, no. 15, pp. 1263-1265, 2014.
[20] C. L. Miao, Q. F. Yu, and H. F. Liang, "Clinical observation of the Huachansu combined with chemotherapy in treatment of advanced non-small cell lung cancer," Chinese Journal of Integrative Medicine, vol. 27, no. 7, pp. 657-658, 2007.

[21] X. D. Miao, H. F. Cao, and W. X. Wang, "Observation on the short-term efficacy of Huachansu combined with TP regimen in treatment of advanced non-small cell lung cancer," Chinese and Foreign Medical Research, vol. 12, no. 28, pp. 130-133, 2014.

[22] R. F. Qi, "Clinical observation of the Huachansu in adjuvant treatment of advanced non-small cell lung cancer," The Chinese and Foreign Health Abstract, vol. 8, no. 12, pp. 80-82, 2011.

[23] S. L. Wang, "Analysis of the therapeutic effect of Huachansu combined with chemotherapy in treatment of advanced nonsmall cell lung cancer," Central Plains Medical Journal, vol. 33, no. 1, p. 70, 2006.

[24] L. G. Xiong and L. L. Li, "Clinical observation of the Huachansu combined with chemotherapy in treatment of advanced nonsmall cell lung cancer," Practical Oncology Journal, vol. 9, no. 4, pp. 294-296, 2005.

[25] X. F. Yang and J. Xi, "Observation of the therapeutic effect of Huachansu combined with chemotherapy in treatment of advanced non-small cell lung cancer," Herald of Medicine, vol. 25, no. 12, pp. 1287-1288, 2006.

[26] H. Y. Yu, S. Y. Gao, and Y. X. Hao, "Study of Huanchansu injection combined with TP regimen in the treatment of patients with advanced non-small cell lung cancer," The Practical Journal of Cancer, vol. 27, no. 1, pp. 55-57, 2012.

[27] J. P. T. Higgins, S. G. Thompson, J. J. Deeks, and D. G. Altman, "Measuring inconsistency in meta-analyses," British Medical Journal, vol. 327, no. 7414, pp. 557-560, 2003.

[28] M. C. McBride, "Bufotenine: toward an understanding of possible psychoactive mechanisms," Journal of Psychoactive Drugs, vol. 32, no. 3, pp. 321-331, 2000. 


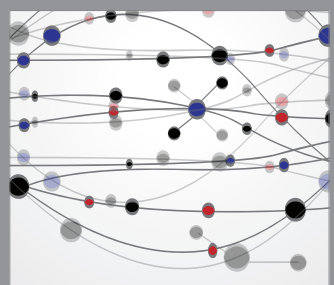

The Scientific World Journal
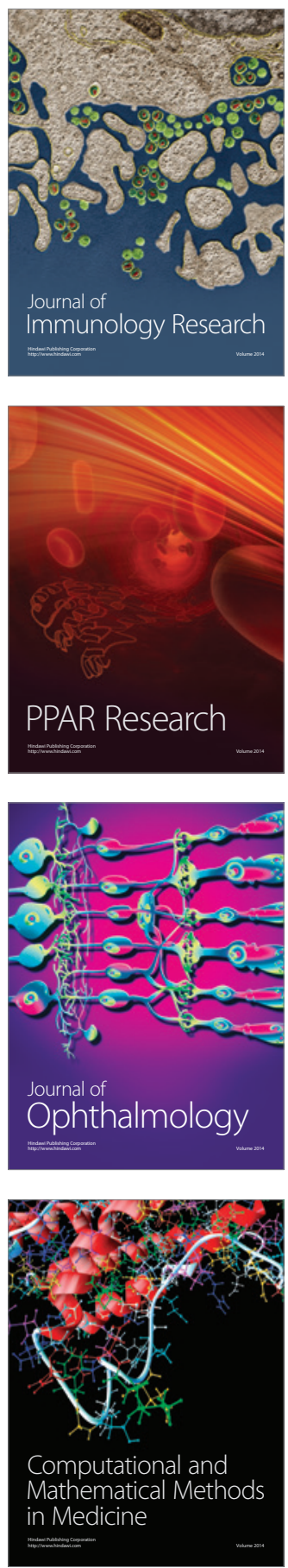

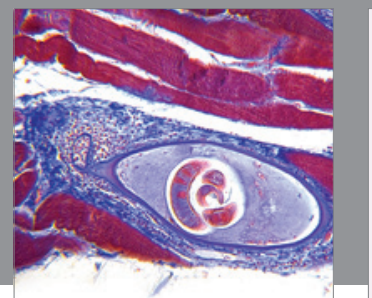

Gastroenterology

Research and Practice
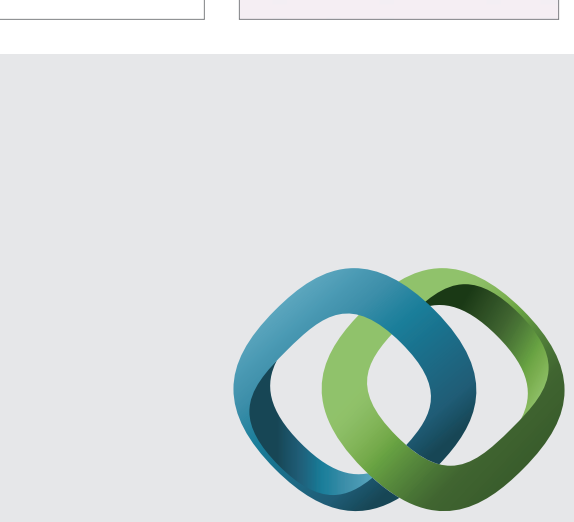

\section{Hindawi}

Submit your manuscripts at

http://www.hindawi.com
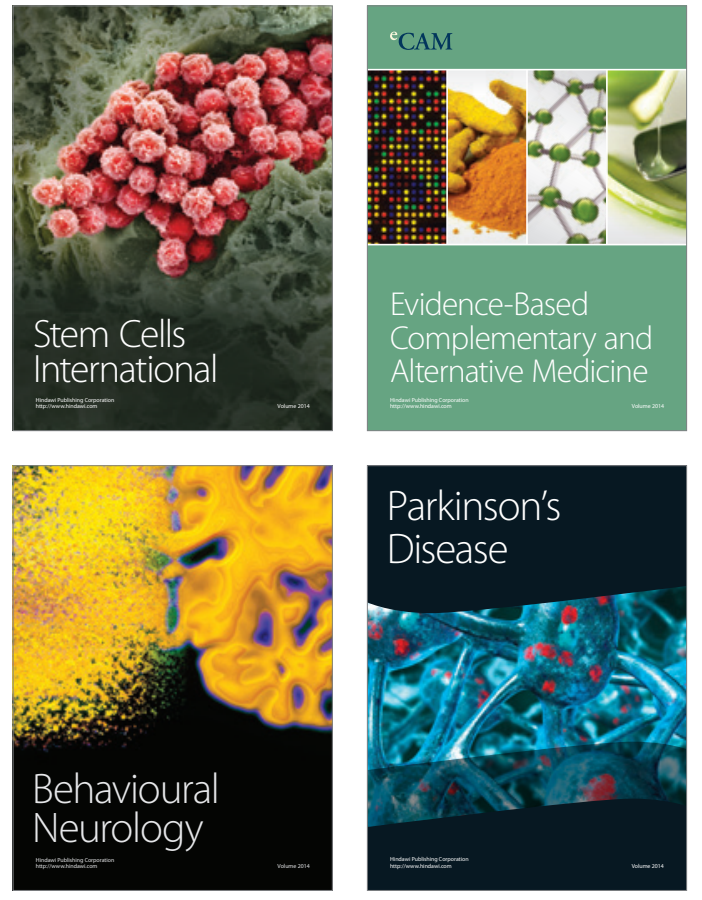
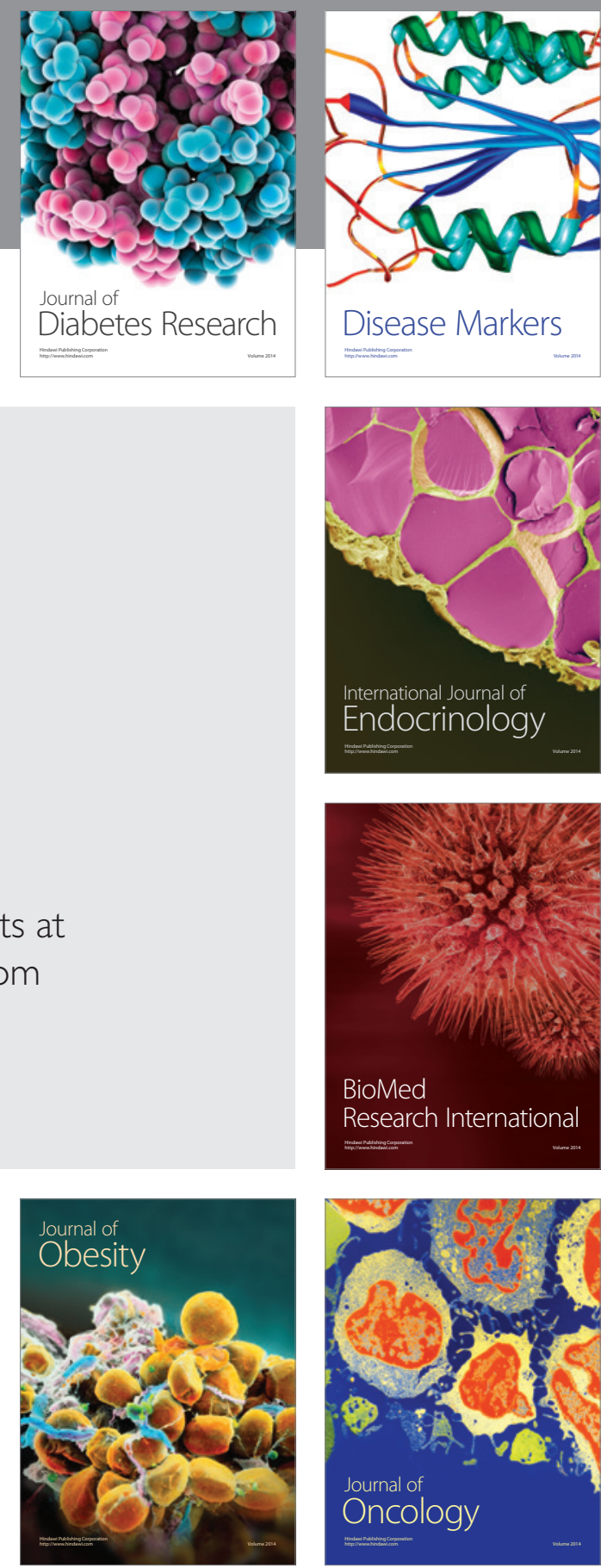

Disease Markers
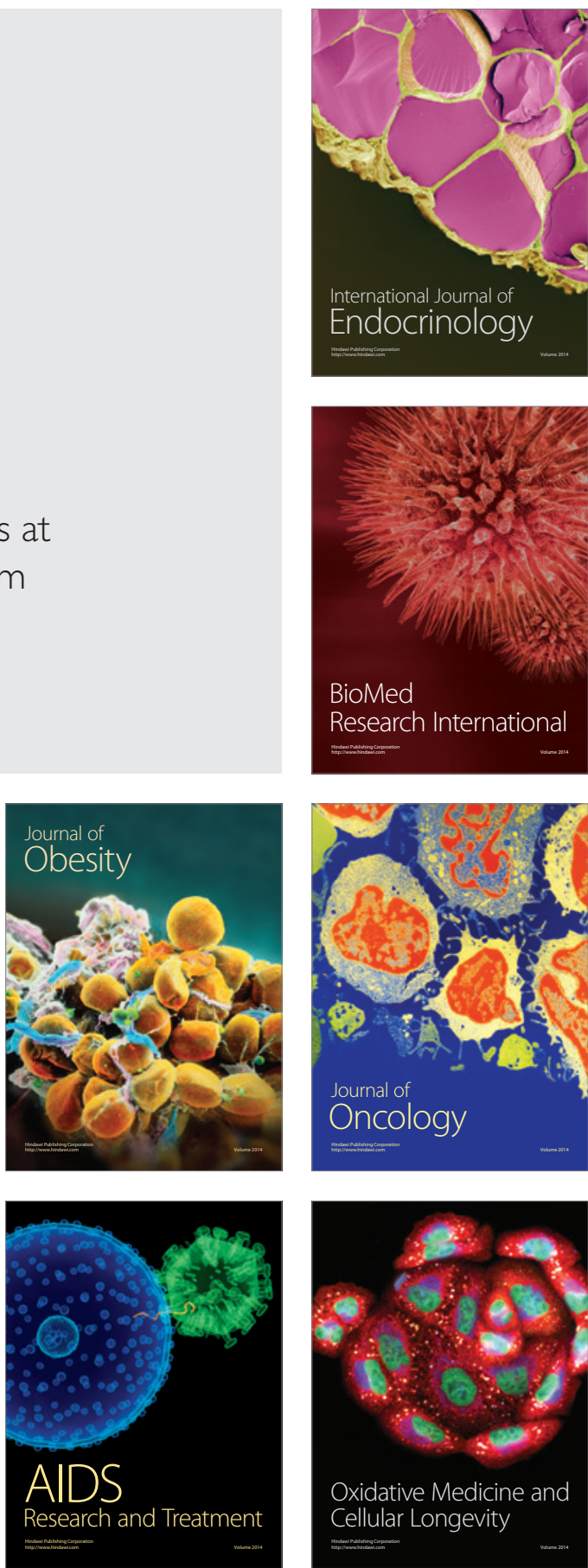